\title{
La valorisation patrimoniale des édifices religieux entre affectation cultuelle exclusive et contractualisation
}

Pierre-Henri Prélot

\section{(Q) OpenEdition \\ Journals}

Édition électronique

URL : http://journals.openedition.org/rdr/816

DOI : $10.4000 /$ rdr.816

ISSN : 2534-7462

Éditeur

Presses universitaires de Strasbourg

Édition imprimée

Date de publication : 3 mai 2017

Pagination : $29-42$

ISBN : 978-2-86820-973-3

ISSN : 2493-8637

\section{Référence électronique}

Pierre-Henri Prélot, « La valorisation patrimoniale des édifices religieux entre affectation cultuelle exclusive et contractualisation », Revue du droit des religions [En ligne], 3 | 2017, mis en ligne le 17 février 2020, consulté le 19 novembre 2020. URL : http://journals.openedition.org/rdr/816 ; DOI : https://doi.org/10.4000/rdr.816

\section{(c) (†) (8)}

La revue du droit des religions est mise à disposition selon les termes de la Creative Commons Attribution - Pas d'Utilisation Commerciale 4.0 International - CC BY-NC 4.0. 


\section{LA VALORISATION PATRIMONIALE DES ÉDIFICES RELLGIEUX ENTRE AFFECTATION CULTUELLE EXCLUSIVE ET CONTRACTUALISATION}

\section{Pierre-Henri PRÉLOT}

Université de Cergy-Pontoise

\section{RÉSUMÉ}

La France est incontestablement l'un des pays d'Europe qui a su le mieux préserver depuis un siècle son patrimoine religieux. Paradoxalement, elle doit cette situation à son régime de séparation qui a garanti aux cultes anciennement reconnus, et en particulier à l'Église catholique, la libre disposition des édifices devenus propriété publique à la Révolution ou au XIX siècle. La prise en charge des réparations par le propriétaire et l'entretien au quotidien par les fidèles ont permis de protéger ces édifices de la transformation, de la destruction ou de la vente. Mais ce modèle est aujourd'hui en crise, parce que les financements font défaut et que la pratique religieuse décline. Il s'agit ici de réfléchir aux conditions juridiques d'une valorisation des édifices religieux susceptible d'assurer leur pérennité dans le respect de l'affectation cultuelle.

\section{ABSTRACT}

France is undoubtedly one of the European countries that has best preserved its religious heritage over the past century. Ironically, it owes this situation to the separation regime which guaranteed the formerly recognized religions, especially the Catholic Church, the free disposal of the church buildings that had become public property during the Revolution or nineteenth century. Repairs carried out by the owner and the routine maintenance provided by the faithful have protected these buildings from transformation, destruction or resale. But this model is in crisis, due to the lack of funding and the decline in religious observance. The purpose of this article is to envisage the legal conditions for an enhancement of religious buildings, likely to ensure their sustainability while respecting the use for worship. 
$\mathrm{L}_{\mathrm{s}}$ es nombreux monuments religieux qui jalonnent le sol de France portent le témoignage d'une histoire plus que millénaire. Qu'ils aient ou non conservé leur fonction d'origine, ils sont notre héritage commun, spirituel bien entendu, mais aussi historique, artistique, culturel, ou encore architectural. Le législateur de 1905 était parfaitement conscient de l'importance de ce patrimoine, et c'est pourquoi l'article $16^{1}$ de la loi ordonna un classement complémentaire des édifices servant à l'exercice du culte qui devait inclure « tous ceux de ces édifices représentant, dans leur ensemble ou dans leurs parties, une valeur artistique ou historique ». Il s'agissait également, pour les biens meubles et immeubles par destination, d'ajouter à la liste de classement établie en application de la loi de 1887 sur les monuments historiques « ceux de ces objets dont la conservation présenterait, au point de vue de l'histoire ou de l'art, un intérêt suffisant », et ce dans un délai de trois ans. Enfin, l'article 16 prévoyait expressément que les immeubles et les objets mobiliers attribués aux associations cultuelles pourraient eux aussi bénéficier du classement. La loi de 1905 n'est donc pas seulement une loi sur le culte, c'est aussi une loi patrimoniale qui fait la transition entre sa devancière de 1887 et la grande loi du 31 décembre 1913 sur les monuments historiques ${ }^{2}$. On ajoutera à cela que même s'il n'énonce aucune exception expresse en ce sens, l'article 2 de la loi de 1905 interdisant les subventions au culte a toujours été compris comme ne faisant pas obstacle à l'entretien par les collectivités publiques du patrimoine religieux classé, et que par ailleurs l'autorisation donnée aux collectivités propriétaires, par la loi du 13 avril 1908, d'engager les dépenses nécessaires pour l'entretien et la conservation des édifices du culte dont elles ont la propriété ${ }^{3}$, a permis depuis un siècle d'assurer la sauvegarde de nombreux monuments anciens.

Mais curieusement, si les parlementaires qui ont fait la séparation avaient parfaitement saisi la grande valeur historique et artistique du patrimoine religieux, la loi qu'ils ont votée ne traite guère que de son usage religieux et néglige en revanche à peu près complètement la question de sa valorisation patrimoniale. Le régime de l'affectation cultuelle exclusive, qui se déduit

1. L'article 16 ne figurait pas dans le projet de loi initial, il a été rajouté par voie d'amendement.

2. On sait que la loi de 1913 a été votée dans un contexte marqué par la campagne publique de Barrès déplorant « la grande pitié des églises de France ». Aujourd'hui, selon le ministère de la Culture, les bâtiments religieux forment $34 \%$ du patrimoine immobilier protégé au titre des monuments historiques, soit plus de 14000 édifices ou parties d'édifices classés ou inscrits (source : FRANCE. SÉNAT, Les collectivités territoriales et le financement des lieux de culte : rapport d'information de M. Hervé Maurey, n 345, 17 mars 2015).

3. Disposition ajoutée à l'article 13 de la loi de 1905. 
du texte de 1905 et de la loi du 2 janvier 1907 concernant l'exercice public des cultes, ne laisse pratiquement aucune place à d'autres usages que ceux purement religieux ${ }^{4}$. Cette exclusivité signifie d'une part que le propriétaire public d'un édifice cultuel ne dispose d'aucun droit d'usage pour son propre compte, et d'autre part que l'affectataire ne peut l'utiliser à des fins autres que religieuses - ce qui serait un motif de désaffectation. On se propose dans les développements qui suivent de rappeler cet état du droit résultant de la loi de 1905 (1). Mais comme on le verra ensuite, la pratique a permis de dégager des solutions nouvelles, de nature conventionnelle, qui autorisent désormais une certaine valorisation patrimoniale des édifices religieux (2).

La présente réflexion laisse de côté les édifices religieux relevant de la propriété privée des organisations religieuses, et qui la plupart du temps appartiennent à une association cultuelle ou diocésaine ${ }^{5}$. Ceux de ces édifices privés, et ils sont nombreux, qui ont été construits après 1905 ne présentent pas, sauf exception, d'intérêt patrimonial particulier ${ }^{6}$. Mais les plus anciens, qui bénéficient d'ailleurs parfois d'un classement ou d'une inscription au titre des monuments historiques, sont souvent d'une grande valeur historique, architecturale et artistique. En ce cas, il est généralement plus aisé pour le propriétaire de valoriser l'édifice dans le respect de son usage religieux. La réflexion laisse également de côté un certain nombre de lieux de culte, moins nombreux, qui appartiennent à des personnes publiques et qui ne relèvent pas du régime de l'affectation de la loi de 1905. L'usage religieux de l'édifice repose alors sur des fondements conventionnels ${ }^{7}$ qui laissent la place à une éventuelle valorisation patrimoniale à l'initiative du propriétaire public. Mais pour n'être pas exhaustif, l'exposé qui suit concerne tout de même l'immense majorité des édifices cultuels qui présentent un intérêt historique, culturel, architectural et artistique, et pour lesquels se pose la question de leur valorisation patrimoniale. Concrètement, ce sont plus de

4. L'article 27 prévoit une exception pour les sonneries civiles des cloches.

5. Il ne s'agit pas d'une obligation au sens légal, le propriétaire d'un édifice religieux peut être une personne physique ou une personne morale d'un autre type, par exemple une association simple de la loi de 1901, une société ou encore une fondation.

6. La plus notable de ces exceptions étant la chapelle de Ronchamp construite par Le Corbusier et classée monument historique.

7. C'est le cas en particulier de l'église abbatiale du Mont-Saint-Michel. V. sur ce point Prélot P.-H., «Affectation culturelle et usage religieux : le cas de l'abbaye du Mont-SaintMichel », in Basdevant-Gaudemet B., Cornu M., Fromageau J. (dir.), Le patrimoine culturel religieux. Enjeux juridiques et pratiques cultuelles, Paris, L'Harmattan, 2006, p. 259-276. Plus généralement, V. sur cette question CE, 19 oct. 1990, n 90346, Association Saint-Pie V et Saint-Pie X de l'Orléanais. 
40000 édifices cultuels qui relèvent du régime de l'affectation légale, pour leur immense majorité des églises communales ${ }^{8}$.

\section{LA VALORISATION PATRIMONIALE, UN IMPENSÉ DE LA LOI DE SÉPARATION}

L'objet de cette première partie est de démontrer que le législateur de 1905 a laissé de côté, alors même qu'il avait parfaitement saisi les enjeux patrimoniaux du texte en discussion, la question de la valorisation du patrimoine religieux. Outre que le caractère exclusif de l'affectation au culte ne laisse aucune place pour d'autres usages, le droit de visiter les édifices n'y est évoqué que de façon très incidente, à la suite d'un amendement parlementaire adopté au forceps.

\subsection{LE CARACTÈRE EXCLUSIF DE L'AFFECTATION DANS LA LOI DE 1905}

La séparation des Églises et de l'État pouvait laisser envisager la restitution à l'Église catholique des édifices nationalisés à la Révolution, et c'est d'ailleurs ce que revendiquaient, comme conséquence de la suppression du budget des cultes, de nombreux partisans du Concordat. À l'extrême gauche, le député-maire de Lyon Victor Augagneur avait lui aussi défendu à la Chambre des députés cette solution séparatiste, mais il n'a pas su convaincre ses collègues anticléricaux qu'il s'agissait de libérer le nu-propriétaire et surtout pas de faire un cadeau à l'Église ${ }^{9}$. Si l'on peut regretter cette solution du point de

8. Sur cette question de la consistance du patrimoine religieux en France, V. France. SÉnat, Les collectivités territoriales et le financement des lieux de culte, op. cit. Selon ce rapport, « $90 \%$ des édifices du culte catholique sont la propriété des communes, alors que ce chiffre ne représente que $12 \%$ pour le culte protestant, $3 \%$ pour le culte juif, et $0 \%$ pour le culte bouddhiste et le culte musulman ». Pour la situation du patrimoine religieux catholique, on pourra également consulter utilement le site http://www.patrimoinereligieux.fr/ [consulté le 13 janv. 2017].

9. Séance du 8 juin 1905, Annales de la Chambre des députés, Paris, 1905 : « Donc ce mot donation a fait un effet considérable et j'ai paru vouloir donner beaucoup. Je ne le crois pas, pour ma part ; car, en théorie, si légalement les édifices du culte appartiennent actuellement aux communes, c'est une propriété de mince valeur, une propriété théorique. Être nu-propriétaire, et, présence d'un usufruitier éternel, c'est une situation de dupe; c'est la réalité de la situation de toutes les communes vis-à-vis des églises. La commune est nue-propriétaire, l'usufruitier ne meurt jamais, et une propriété de ce genre ne comporte que des charges. Personne n'aurait l'idée d'acheter en viager la propriété d'un monsieur qui ne devrait jamais mourir. (Sourires.) C'est donc une propriété de nulle valeur à l'heure présente. » Plus attendus parce qu'en défense de l'Église catholique, 
vue de la logique séparatiste, il y a tout lieu de penser à plus d'un siècle de distance, et à la lumière des expériences étrangères, que c'est le maintien de la propriété publique qui a heureusement permis de préserver l'intégrité du patrimoine religieux ${ }^{10}$. Le législateur a par ailleurs exclu la solution inverse de la désaffectation de tous les édifices, et le retour en pleine possession et jouissance au propriétaire public ${ }^{11}$. La solution du bail emphytéotique, à laquelle il est aujourd'hui recouru pour les constructions de nouveaux lieux de culte, a également été écartée après discussion ${ }^{12}$. Quant au projet de loi du gouvernement, il consistait après deux années de statu quo à imposer au propriétaire public une obligation de location décennale des édifices aux associations cultuelles, solution dont l'avantage était de permettre un retour progressif au propriétaire. Mais les débats parlementaires ont fait prendre conscience des difficultés pratiques de cette solution ${ }^{13}$ et la commission a fini par se rallier au principe d'une mise à disposition gratuite et permanente des édifices pour la pratique du culte. Ce régime d'affectation cultuelle figure à l'article 13 de la loi, énonçant que les édifices « seront laissés gratuitement à la disposition des établissements publics du culte, puis des associations appelées à les remplacer $»$. Compte tenu de l'absence d'anticipation de la solution finalement choisie, on ne retrouve pas dans les débats parlementaires, ni a fortiori dans le rapport de la commission parlementaire présidée par Ferdinand Buisson, de réflexion concernant l'étendue de cette mise à disposition, mais celle-ci a immédiatement été comprise dans un sens doublement exclusif. D'une part, l'édifice devait être laissé à l'entière disposition de l'affectataire religieux, sans que la collectivité publique puisse en faire un

des amendements de Jules Dansette et Jules Auffray demandent également le retour des édifices à leur propriétaire originaire.

10. Selon un document de 2016 de la Conférence des évêques de France, seules 255 églises (sur un total de 42258 églises affectées) ont été désaffectées ou vendues depuis 1905. Sur ce total, 22 ont été vendues et/ou reconverties: CEF, État des lieux des églises en France, sept. 2016.

11. V. en ce sens la proposition Allard et autres du 8 juin 1905 : elle n'excluait pas la location des édifices à des fins cultuelles, mais pour une durée maximale de 4 ans, et à un prix qui ne pouvait être inférieur à $5 \%$ de la valeur de l'immeuble.

12. Le système du bail emphytéotique a été proposé par Étienne Flandin.

13. «Des explications échangées hier à la tribune, il nous a paru résulter que la Chambre désirait à la fois éviter aux conseils municipaux les difficultés de négociations délicates avec les associations cultuelles pour la location des églises appartenant aux communes et en même temps assurer à ces associations une possession des édifices assez prolongée et assez stable. [...] Le système que nous lui proposons consiste à laisser aux associations cultuelles la jouissance illimitée et gratuite des édifices dont la propriété est affirmée par l'article $10 \mathrm{au}$ profit, selon les cas, de l'État, des départements ou des communes. » : Aristide Briand, séance du 9 juin 1905. 
quelconque usage. Une exclusivité que matérialisait formellement la règle selon laquelle le maire ne détient pas en propre les clefs de l'église, sauf en l'absence d'accès indépendant au clocher ${ }^{14}$. D'autre part, l'affectataire religieux ne pouvait utiliser l'édifice dans un but autre que religieux, le fait de détourner un édifice de sa destination constituant un motif de désaffectation.

Cette exclusivité de l'affectation s'est d'autant mieux imposée qu'elle créait des obligations croisées liant l'un envers l'autre chacun des partenaires public et religieux. Elle a incontestablement joué un rôle pacificateur au lendemain de la séparation. C'est pourquoi la loi du 2 janvier 1907, prenant acte du refus de l'Église catholique de constituer des associations pour l'exercice du culte, a choisi de prolonger le dispositif en laissant les édifices «à la disposition des fidèles et des ministres du culte pour la pratique de leur religion ». Cette économie générale de la loi présentait l'avantage de protéger les lieux de culte contre les usages intempestifs de toute sorte, mais elle n'a pas permis en revanche de fixer les conditions de leur valorisation artistique et culturelle, ou encore de leur exploitation touristique, qui s'inscrivait en contradiction avec l'usage purement religieux qui en était prescrit. Or non seulement une telle contradiction est un contresens, dans la mesure où la valorisation patrimoniale et l'affectation religieuse se complètent plus qu'elles ne se contredisent la plupart du temps, mais de surcroît cette valorisation requiert en puissance la contribution simultanée du propriétaire public et de l'affectataire religieux. C'est pourquoi, à plus d'un siècle de distance, la valorisation patrimoniale apparaît comme un grand impensé de la loi de Séparation.

\subsection{LE RÉGIME MINIMALISTE DES VISITES}

Il a fallu une fois encore que le législateur se fasse forcer la main pour consentir finalement à traiter de la question des visites ${ }^{15}$. De haute lutte, le socialiste Gustave Rouanet a réussi à obtenir l'ajout, à l'article 17, d'un amendement énonçant que «la visite des édifices et l'exposition des objets mobiliers classés seront publiques : elles ne pourront donner lieu à aucune taxe ni redevance ». Ce postulat généreux de gratuité d'accès à la culture sera

14. Les cloches doivent également pouvoir servir aux sonneries civiles.

15. Sur le régime des visites, V. FORNEROD A., «Les prémices d'une réforme de la protection des monuments historiques : la loi de 1905 », in BADY J.-B. et al. (dir.), 1913, genèse d’une loi sur les monuments historiques, Paris, La Documentation française, 2013, p. 70-78. Plus généralement, on pourra à propos de la valorisation patrimoniale des édifices cultuels consulter la thèse consacrée par A. Fornerod à cette question : Le régime juridique du patrimoine religieux, Paris, L'Harmattan, 2013. 
atténué ponctuellement par la loi du 31 décembre 1913 sur les monuments historiques, qui autorise (art. 25) la perception d'un droit de visite des objets mobiliers classés, mais uniquement «à raison des charges supportées par les communes et départements propriétaires pour l'exécution des mesures de garde et de conservation ». Ainsi qu'a pu le souligner le commissaire du gouvernement Schwartz dans ses conclusions sur l'arrêt Abbé Chalumey de 1994, "le motif légal posé par la loi du 31 décembre 1913 à l'institution d'un droit de visite est la compensation des frais de garde et de conservation des objets mobiliers supportés par la commune », il n'est pas de « recueillir des fonds pour entretenir les bâtiments ${ }^{16}$. Le principe de la gratuité intégrale est préservé en revanche pour les édifices appartenant à l'État et donc pour les cathédrales.

En ce qui concerne plus précisément les édifices de l'État, la loi de finances du 31 décembre 1921 (art. 118) autorise l'administration des Beaux-arts à percevoir un droit d'entrée pour la visite des musées, collections et monuments, mais ainsi que le précise ce même texte, « ces dispositions ne s'appliquent pas aux édifices visés par l'article $17 £ 6$ de la loi du 9 décembre 1905 ». Autrement dit, la situation créée par la législation séparatiste en matière de valorisation du patrimoine religieux peut se résumer de la façon suivante :

- Seules les visites, "parent pauvre ${ }^{17}$ de la législation sur le patrimoine, sont évoquées par la loi de Séparation. Encore ne le sont-elles que pour énoncer un principe général de gratuité, atténué par la loi de 1913 sur les monuments historiques, et que vient conforter au contraire, pour les cathédrales de l'État - c'est-à-dire pour les monuments les plus susceptibles d'attirer les touristes - la loi de 1921. Outre que la gratuité empêche de recueillir des fonds pour l'entretien - de plus en plus coûteux - des édifices, le régime des visites présente un caractère subsidiaire, puisqu'en application du décret du 16 mars 1906 pris pour l'exécution de la loi de Séparation, ce sont les associations cultuelles qui fixent, dans le but légitime de préserver l'affectation cultuelle, « les jours et heures auxquels auront lieu [...] la visite des édifices et l'exposition des objets mobiliers classés », et ce « sous réserve de l'approbation du préfet » (art. 29) ${ }^{18}$;

16. CE, 4 nov. 1994, n 135842, Abbé Chalumey, RFDA 1995, p. 991 : cité par Fornerod A., Le régime juridique du patrimoine religieux, op. cit., p. 384.

17. La formule est d'Anne Fornerod : «Les prémices d'une réforme de la protection des monuments historiques : la loi de $1905 »$, op. cit., p. 76.

18. On sait que l'Église catholique n'ayant pas créé les associations cultuelles prévues par la loi de 1905, l'article 29 du décret de 1906 ne s'applique pas dans les édifices du culte catholiques, c'est-à-dire en réalité l'immense majorité du patrimoine religieux public. 
- Les autres formes de valorisation patrimoniale telles que concerts, spectacles ou expositions sont complètement ignorées, alors même qu'elles vont connaître un développement important au fil du temps ;

- Hors la question des visites, les compétences respectives de l'autorité publique propriétaire et de l'affectataire religieux en matière de valorisation patrimoniale ne sont en aucune façon définies. Le propriétaire n'a pas le droit d'utiliser l'édifice à son propre avantage et l'affectataire ne peut le faire qu'à des fins religieuses.

\section{L'ÉMERGENCE D'UN MODÈLE CONVENTIONNEL}

À défaut de pouvoir trouver dans la loi de 1905 un fondement pour la mise en ouvre d'une politique patrimoniale, c'est sous la condition de l'accord du clergé affectataire, aujourd'hui formalisé dans des conventions, que les collectivités propriétaires ont pu engager des actions de valorisation de leurs édifices religieux.

\subsection{LES PRATIQUES DE VALORISATION CULTURELLE}

Cet état lacunaire du droit en matière de valorisation patrimoniale n'est apparu qu'assez tardivement, et c'est la chute massive de la pratique religieuse en même temps que l'intérêt croissant des touristes pour le patrimoine historique religieux, combinés avec la nécessité de plus en plus pressante d'en financer l'entretien, qui ont permis d'en prendre l'exacte mesure. Au fil du temps, des solutions ponctuelles ont pu être trouvées afin de permettre une certaine valorisation des édifices. La plus remarquable a consisté, lorsque la configuration des lieux le permettait, en la division spatiale de l'édifice cultuel entre les parties utilisées pour le culte et celles qui, dépourvues de tout usage religieux, étaient susceptibles d'être exploitées par le propriétaire sans préjudice pour les fidèles. Un tel partage n'était nullement envisagé par le législateur, mais il a été entériné implicitement par le Conseil d'État dans son fameux arrêt Carlier de $1949^{19}$. Dans cet arrêt, le Conseil d'État annule, en raison de son caractère disproportionné, une décision du directeur général des Beaux-arts interdisant à un photographe professionnel « l'accès des parties de la cathédrale de Chartres où n'est célébré aucun office du culte». C'est sur ce fondement d'un partage de l'espace qu'a pu être organisée la visite de

19. CE, ass., 18 nov. 1949, n 77441, Sieur Carlier, Rec. CE, p. 490. 
certaines parties des édifices religieux, telles que les tours de Notre-Dame de Paris. Encore la redevance prélevée pour les visites restait-elle irrégulière au regard du principe de gratuité résultant, pour les cathédrales de l'État, de la loi de 1921. Ce principe de division spatiale comme fondement d'une valorisation autonome des édifices cultuels par le propriétaire a été confirmé de façon expresse par le Conseil d'État dans un arrêt de 2012 qui consacre la théorie dite de la dissociabilité fonctionnelle. Dans cet arrêt qui concernait le toit-terrasse de l'Église des Saintes-Maries-de-la-Mer ${ }^{20}$, le Conseil d'État a considéré que l'affectation au culte «s'applique à l'ensemble d'un édifice cultuel, y compris ses dépendances nécessaires, fonctionnellement indissociables de l'édifice cultuel », mais que toutefois

« il en va autrement d'aménagements qui [...] doivent être regardés, compte tenu notamment de leurs caractéristiques propres et de la possibilité d'y accéder sans entrer dans l'édifice cultuel, comme fonctionnellement dissociables de cet édifice; que la commune peut, sans avoir à recueillir l'accord préalable du desservant de l'église, organiser des visites de tels aménagements; qu'il lui appartient de veiller à ce que les modalités d'organisation de celles-ci ne conduisent pas à perturber l'exercice du culte à l'intérieur de l'édifice et soient compatibles avec l'affectation de l'édifice sur lequel les aménagements visités sont situés».

À la suite de l'arrêt Carlier, on a voulu voir dans le pouvoir de police $\mathrm{du}$ propriétaire public un fondement légal ${ }^{21}$ pour l'accueil et la prise en charge des visiteurs à l'intérieur des édifices, et par voie de conséquence pour l'organisation des activités profanes de toute sorte. Mais outre que le pouvoir de police n'est pas en soi un fondement de compétence matérielle, cette vision était contredite par le maintien, après la séparation, du pouvoir de police du desservant à l'intérieur des lieux de culte ${ }^{22}$.

Le recours à la théorie de l'accessoire a également permis le développement, au bénéfice cette fois de l'affectataire, de certains usages non directement religieux. Si l'article 19 de la loi de 1905 autorise les quêtes et collectes pour les frais du culte à l'intérieur des édifices légalement affectés, de même que la perception de rétributions pour les cérémonies et services religieux,

20. CE, 20 juin 2012, n 340648, Commune des Saintes-Maries-de-la-Mer, Rec. CE, p. 247.

21. Selon l'article 97 de la loi municipale du 5 avril 1884, « La police municipale [...] comprend notamment [...] le maintien du bon ordre dans les endroits où il se fait de grands rassemblements d'hommes, tels que les foires, marchés, réjouissance et cérémonies publiques, spectacles, jeux, cafés, églises et autres lieux publics ».

22. V. sur ce point CE, 3 mai 1918, n 58743, Abbé Piat. 
elle n'autorise pas en revanche la vente de livres et de guides, de cartes postales et d'objets religieux divers (médailles, chapelets, DVD, etc.). De la même manière, les concerts de musique religieuse qui sont régulièrement organisés dans les églises à l'initiative du clergé affectataire n'ont aucun fondement légal dans le texte de 1905. Mais ces activités, qui sont considérées par l'Église catholique comme un prolongement de la pastorale, font l'objet d'une tolérance de la part de l'autorité publique en vertu de la théorie dite de l'accessoire fondée sur une conception élargie de la notion de culte. Aux lendemains de la séparation, une telle pratique n'allait évidemment pas de soi. La circulaire du ministre de l'Instruction publique, des Beaux-arts et des Cultes en date du $1^{\text {er }}$ décembre 1906, relative aux conditions d'exercice du culte public à défaut d'associations cultuelles, prescrit que l'affectataire « n'aura pas qualité pour percevoir des rétributions à raison de l'usage par des tiers de l'église ou des objets qui y sont contenus et qui appartiennent à l'État ou aux communes ou auront appartenu à la fabrique supprimée. Il aura seulement la faculté de recueillir des offrandes à l'occasion des actes de son ministère $»^{23}$. Ainsi que l'établissent les débats parlementaires de la loi de 1905, le principe de la gratuité des visites devait s'imposer à l'affectataire tout autant qu'au propriétaire.

À défaut de pouvoir disposer d'un support légal pour leurs projets de valorisation des édifices, les collectivités publiques propriétaires ont cherché à leur donner un fondement consensuel, en sollicitant l'accord de l'affectataire religieux. Cette exigence d'accord préalable était en cohérence avec le droit canonique de l'Église catholique, qui l'a de ce fait accueillie très favorablement, puisqu'elle permettait à la fois de protéger la pratique religieuse et de préserver «la sainteté du lieu ${ }^{24}$.

C'est le Conseil d'État qui allait transposer dans le droit de la séparation les exigences canoniques. Il l'a fait tout d'abord à propos du droit de visite prévu à l'article 17 de la loi de $1905^{25}$. Dans un arrêt Abbé Chalumey du 4 novembre $1994^{26}$, il a considéré que le caractère exclusif de l'affectation

23. Circulaire du ministre de l'Instruction publique, des Beaux-arts et des Cultes du $1^{\text {er }}$ décembre 1906 relative aux conditions d'exercice du culte public à défaut d'associations cultuelles, JO, 2 déc. 1906, p. 7979 : cité par FORNEROD A., Le régime juridique du patrimoine religieux, op. cit., p. 378.

24. Selon le canon 1210, «Ne sera admis dans un lieu sacré que ce qui sert ou favorise le culte, la piété ou la religion, et y sera défendu tout ce qui ne convient pas à la sainteté du lieu. Cependant l'ordinaire peut permettre occasionnellement d'autres usages qui ne soient pourtant pas contraires à la sainteté du lieu ».

25. Complété par l'article 25 de la loi du 31 décembre 1913 sur les monuments historiques.

26. Décision précit. 
cultuelle impliquait nécessairement l'accord du desservant pour l'organisation des visites par le conseil municipal ${ }^{27}$. Cette solution reproduisait, mais en la retournant, la formule de l'article 29 du décret du 16 mars $1906^{28}$. Une dizaine d'années plus tard, la même solution a été étendue par l'ordonnance de référé du 25 août 2005 Commune de Massat ${ }^{29}$ à l'ensemble des activités non religieuses susceptibles d'être organisées dans un lieu public par son propriétaire, remettant en cause le principe de l'indisponibilité des lieux de culte, auquel le législateur républicain avait pourtant conféré un caractère d'ordre public. Dans cette affaire, le juge des référés énonce « que les prérogatives patrimoniales de la commune se trouvent subordonnées au respect de l'affectation cultuelle, qui consiste dans l'occupation de l'édifice par le curé et ses fidèles et dans les pouvoirs reconnus au prêtre d'en régler l'usage et l'agencement cultuel; que la liberté de culte est une liberté fondamentale [...] qui comprend notamment la possibilité d'utiliser un lieu exclusivement à l'usage religieux et très accessoirement aux usages autorisés par le desservant seul, pour préserver le caractère sacré du lieu ». Si la rédaction de cette ordonnance est, en dépit de son apparente précision, particulièrement ambiguë, en revanche les conclusions qui s'en déduisent sont très claires. En effet, dès lors qu'un édifice cultuel est affecté « exclusivement » à l'usage religieux, et pour peu que les mots aient un sens, il ne peut y avoir dans cet édifice d'autre activité que religieuse. La théorie de l'accessoire à laquelle fait implicitement référence le juge (" accessoirement») ne fait d'ailleurs pas exception, dans la mesure où précisément les activités relevant de l'accessoire (un concert de musique sacrée par exemple) font partie du culte entendu au sens large.

27. "Considérant qu'en vertu des dispositions combinées des lois susvisées du 9 décembre 1905 et du 2 janvier 1907, en l'absence d'associations cultuelles et d'actes administratifs attribuant la jouissance des églises et des meubles les garnissant, ces biens sont laissés à la disposition des fidèles et des desservants ; que leur occupation doit avoir lieu conformément aux règles d'organisation générale du culte et que les ministres du culte occupant les édifices sont chargés d'en régler l'usage de manière à assurer aux fidèles la pratique de leur religion; qu'il suit de là qu'en décidant d'instituer, en application des dispositions de l'article 25 de la loi susvisée du 31 décembre 1913, un droit de visite des objets mobiliers classés exposés dans l'église Saint-Pierre de Baume-les-Messieurs sans avoir recueilli l'accord du desservant, le conseil municipal de ladite commune a porté atteinte aux droits qui sont reconnus à ce dernier pour réglementer l'usage des biens laissés à la disposition des fidèles par les lois susvisées des 9 décembre 1905 et 2 janvier 1907 ; que la décision d'instituer un tel droit de visite étant ainsi entachée d'illégalité, les décisions prises pour son application doivent être annulées. »

28. Comme on l'a dit précédemment, le décret confiait aux associations cultuelles le soin d'organiser le régime des visites, sous réserve de l'approbation du préfet. L'arrêt Abbé Chalumey exige quant à lui l'accord du desservant à propos de la réglementation des visites par le conseil municipal, pour les lieux de culte ne relevant pas d'une association cultuelle.

29. CE, ord. réf., 25 août 2005, n 284307, Commune de Massat. 
Mais en ajoutant que les usages non religieux ${ }^{30}$ doivent être « autorisés par le desservant seul », dans un litige où les animations envisagées n'avaient pas le moindre caractère religieux, l'ordonnance érige en principe général le fait que tous les usages envisagés par la collectivité propriétaire deviennent désormais possibles, pour autant qu'ils sont autorisés par le desservant. En l'espèce, il s'agissait pour la commune d'organiser dans une chapelle non désaffectée des manifestations diverses, à savoir une représentation théâtrale, une exposition et une conférence-débat sur le $60^{\mathrm{e}}$ anniversaire de la libération des camps de concentration nazis, une exposition de peinture et enfin un concert de musique des Andes, autant d'activités qui de près ou de loin n'ont rien à voir avec l'exercice du culte. De fait le desservant fraîchement nommé s'y est opposé, mais s'agissant d'une chapelle qui n'était pratiquement plus utilisée pour les offices, rien n'interdit de penser qu'une autorisation au moins partielle aurait été accordée dans un contexte moins conflictuel. Finalement, comme le rappelle l'ordonnance, la seule limite qui s'impose au desservant tient dans l'obligation - que lui fait également le droit canon - de "préserver le caractère sacré du lieu ». Encore cette appréciation comportet-elle dans la pratique pastorale une certaine part de subjectivité.

\subsection{LA CONSÉCRATION LÉGISLATIVE D'UNE LOGIQUE CONVENTIONNELLE}

D'une logique d'agrément, il restait un pas à franchir pour aboutir à une logique conventionnelle, où la valorisation patrimoniale des édifices résulterait de l'accord commun du propriétaire et de l'affectataire. C'est cette transition qu'opère en 2006 l'article L. 2124-31 du Code général de la propriété des personnes publiques aux termes duquel :

« Lorsque la visite de parties d'édifices affectés au culte, notamment de celles où sont exposés des objets mobiliers classés ou inscrits, justifie des modalités particulières d'organisation, leur accès est subordonné à l'accord de l'affectataire. Il en va de même en cas d'utilisation de ces édifices pour des activités compatibles avec l'affectation cultuelle. L'accord précise les conditions et les modalités de cet accès ou de cette utilisation. Cet accès ou cette utilisation donne lieu, le cas échéant, au versement d'une redevance domaniale dont le produit peut être partagé entre la collectivité propriétaire et l'affectataire».

Ainsi qu'on le remarque immédiatement, le mot accord est utilisé ici dans son double sens d'agrément unilatéral (de l'affectataire) mais aussi de

30. Ce que l'ordonnance appelle les «prérogatives patrimoniales de la commune». 
convention matérialisant des engagements réciproques, et prévoyant le cas échéant le partage des recettes. Ce prolongement conventionnel de l'autorisation préalable présente donc un double aspect. D'une part, il permet de préciser les conditions concrètes de mise en œuvre des activités de valorisation, et par là de protéger l'usage religieux. D'autre part, il comporte une dimension incitative, en autorisant le versement à l'affectataire religieux d'une partie de la redevance domaniale. On remarque que le Conseil d'État a consacré en 2011 une logique conventionnelle assez similaire, dans son arrêt Commune de Trélazé $e^{31}$, à propos de l'installation aux frais de la commune propriétaire d'un orgue dans une église. Alors que la cour administrative d'appel avait estimé classiquement « que tout équipement installé dans une église ne peut qu'être exclusivement affecté à l'exercice d'un culte », le Conseil d'État au contraire a renvoyé aux « conditions convenues entre le desservant et la commune ", l'orgue ayant vocation à être également utilisé par cette dernière « afin notamment de développer l'enseignement artistique et d'organiser des manifestations culturelles » dans les conditions définies par une convention passée avec l'affectataire.

Autrement dit, c'est une logique que l'on est tenté de qualifier de néo-concordataire qui est aujourd'hui mise en ouvre pour résoudre les apories de la loi de 1905 en matière de valorisation du patrimoine ${ }^{32}$. Cette logique présente incontestablement un certain nombre d'avantages, le principal étant qu'elle préserve l'affectation religieuse des lieux de culte et qu'elle interdit de les transformer en de vastes salles de spectacle ouvertes au jour le jour à toutes sortes d'usages. Mais la contrepartie d'une telle logique est que la valorisation patrimoniale des édifices religieux par le propriétaire reste suspendue à la réalisation d'une condition subjective qui est l'accord préalable du desservant, sans lequel rien n'est possible. Or si la rareté des contentieux atteste que dans l'immense majorité des cas les accords nécessaires parviennent à être trouvés, notamment parce que l'Église a pris conscience des enjeux financiers et qu'elle est sensible à l'intérêt de la population pour le patrimoine religieux, il reste que le desservant peut à tout moment s'opposer à un projet, y compris dans des églises où le culte n'est pratiquement plus célébré, et sans avoir besoin d'invoquer le moindre motif puisque la loi lui maintient l'usage intégral des édifices. Mais s'agissant du Concordat, on se

31. CE, 19 juill. 2011, n 308544, Commune de Trélazé.

32. Pour une autre illustration du recours à la logique conventionnelle en matière de patrimoine religieux, V. également PRÉLOT P.-H., « Affectation culturelle et usage religieux : le cas de l'abbaye du Mont-Saint-Michel ", in Basdevant-Gaudemet B., Cornu M., Fromageau J. (dir.), op. cit., p. 259-276. 
rappelle que les articles organiques avaient rétabli la vieille procédure de l'appel comme d'abus, en autorisant toute personne intéressée à saisir le Conseil d'État «dans tous les cas d'abus de la part des supérieurs et autres personnes ecclésiastiques » (art. 6). Symétriquement, le Conseil d'État pouvait être saisi en cas d'« atteinte à l'exercice public du culte et à la liberté que les lois et les règlements garantissent à ses ministres ». C'est cette logique d'arbitrage entre deux partenaires en charge d'intérêts légitimes et très largement complémentaires, mais qui peuvent être amenés à se contredire ponctuellement, qui manque au dispositif actuellement en place. Il ne s'agit pas seulement, à travers une telle faculté d'arbitrage, de prévenir les éventuels abus en permettant leur sanction, mais plus fondamentalement d'affirmer cet authentique droit à la valorisation patrimoniale des édifices religieux que la loi de 1905 n'avait pas cru devoir consacrer afin de préserver les droits exclusifs de l'affectataire religieux. Quoi qu'il en soit, le succès du modèle conventionnel que définit le Code général de la propriété des personnes publiques reste tributaire de la pérennité du modèle dit séparatiste de l'affectation cultuelle exclusive, dont il est un avatar. Or aujourd'hui c'est le recul constant, dans la plupart des églises, de la pratique religieuse, devenue de plus en plus occasionnelle, bien plus que les impératifs de la valorisation patrimoniale, qui conduit à s'interroger sur la pérennité des équilibres définis en 1905. Il serait cependant illusoire de penser que ce sont les touristes qui à l'avenir pourraient remplacer les fidèles, par substitution d'une affectation culturelle à l'affectation cultuelle traditionnelle. Leur sort est étroitement solidaire et il est lié pour longtemps encore. 